\title{
Electronic Supplement for the Article: Observation of Cirrus Clouds with GLORIA during the WISE Campaign: Detection Methods and Cirrus Characterization
}

\author{
I. Bartolome ${ }^{1}$, R. Spang ${ }^{1}$, J. Ungermann ${ }^{1}$, S. Griessbach ${ }^{2}$, M. Krämer ${ }^{1}$, M. Höpfner ${ }^{3}$, and M. Riese ${ }^{1}$ \\ ${ }^{1}$ Institute für Energie and Klimaforschung (IEK-7), Forschungszentrum Jülich GmbH, 52428 Jülich, Germany \\ ${ }^{2}$ Jülich Supercomputing Centre (JSC), Forschungszentrum Jülich GmbH, 52428 Jülich, Germany \\ ${ }^{3}$ Institute of Meteorology and Climate Research, Karlsruhe Institute of Technology, 76021 Karlsruhe, Germany
}

Correspondence: Irene Bartolome Garcia (i.bartolome@fz-juelich.de)

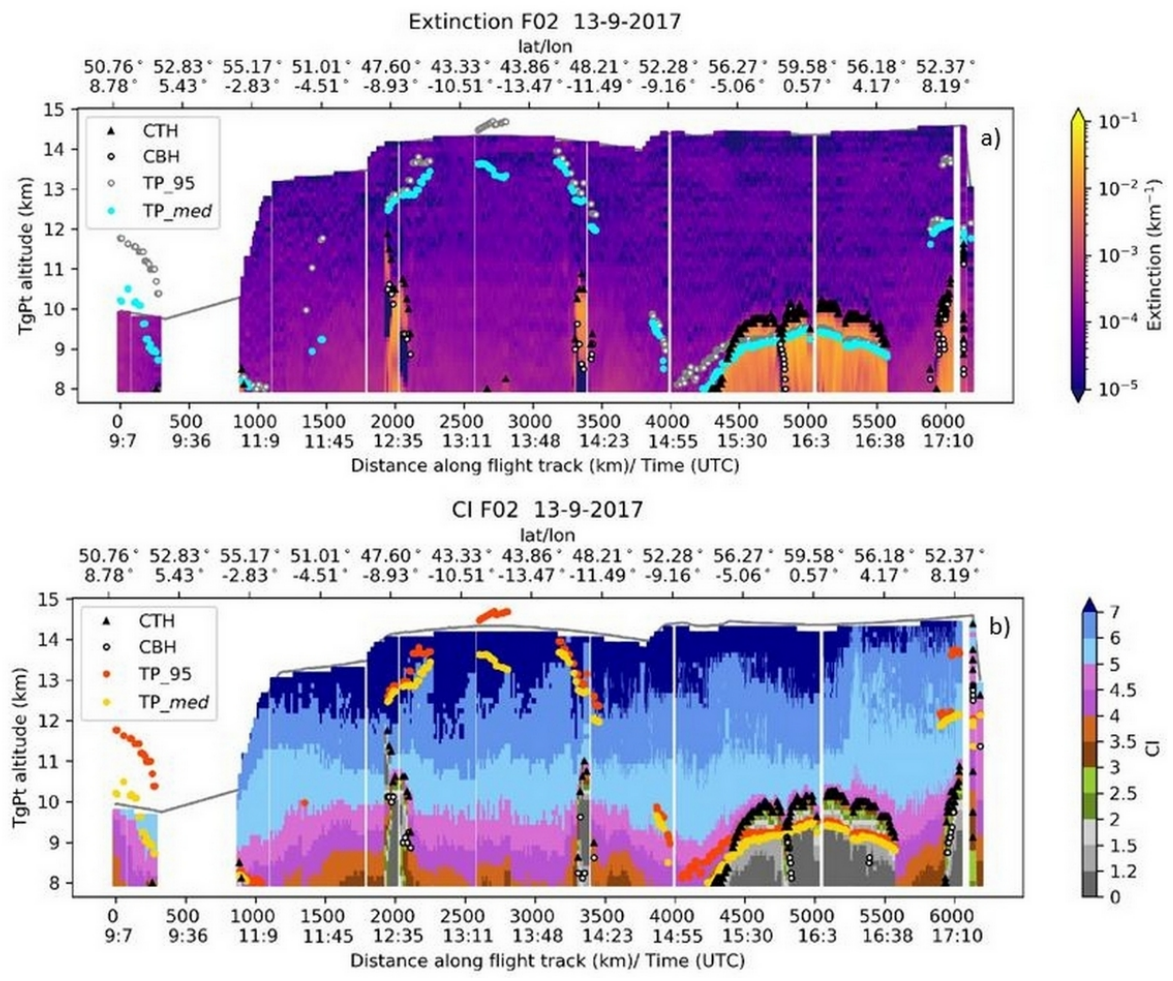

Figure S1. Cross-sections of extinction (a) and cloud index (b) for flight 2 of the WISE campaign. The results are restricted to levels below flight path. (a) Color code for extinction in $\mathrm{km}^{-1}$. Orange-pink colors indicate the presence of clouds; (b) color code for CI. Depending on the altitude, CI values below 2 to 5 (colors from grey to pink) indicate the presence of clouds. Median tropopause (TP $\mathrm{med})$ and the percentile 95 of the tropopause $\left(\mathrm{TP}_{95}\right)$ are represented with orange and yellow circles, respectively. Cloud top height (CTH) and cloud bottom height $(\mathrm{CBH})$ are represented with a black triangle and with a white circle, respectively. The altitude of the tangent points (TgPt) is the $\mathrm{y}$ axis. The white areas in both cross-sections correspond to a first filtering of optically thicker regions $(\mathrm{CI}<2)$. 


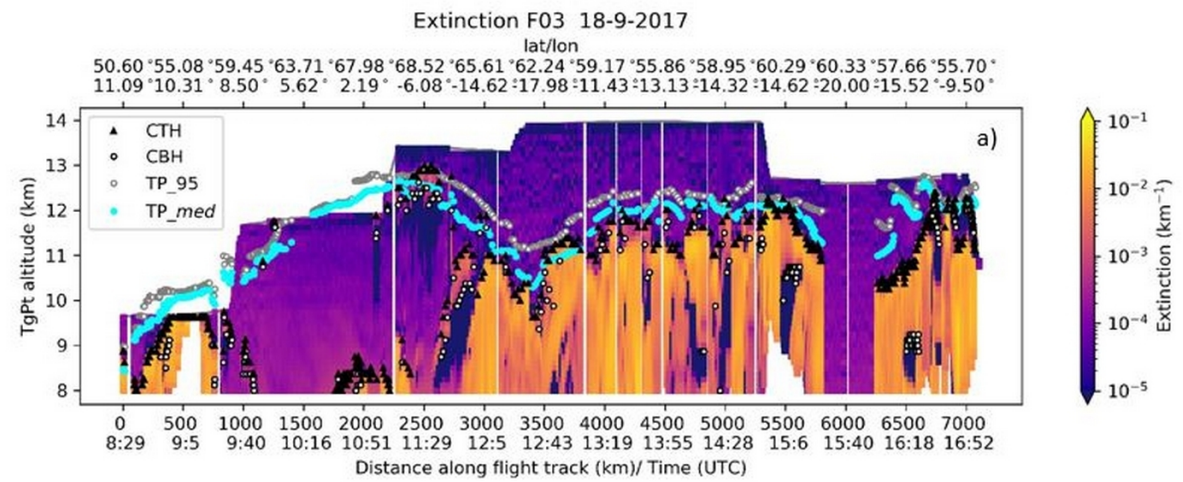

\section{$\mathrm{Cl} \mathrm{F03} \mathrm{18-9-2017}$}

$50.60 * 55.08 * 59.45 * 63.71 * 67.98 * 68.52 * 65.61 * 62.24 * 59.17 \cdot 55.86 * 58.95 * 60.29 * 60.33 * 57.66 * 55.70$.

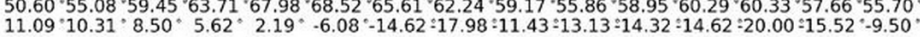

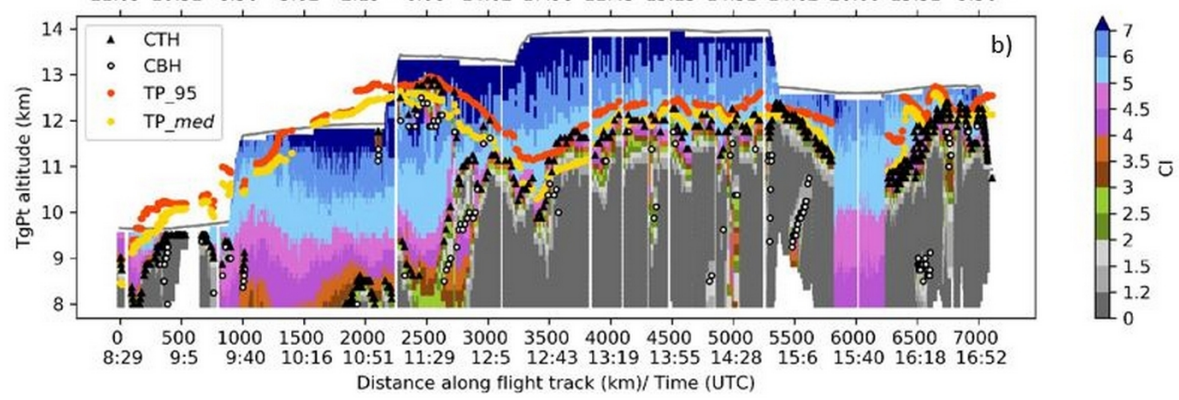

Figure S2. Cross-sections of extinction (a) and cloud index (b) for flight 3 of the WISE campaign. The results are restricted to levels below flight path. (a) Color code for extinction in $\mathrm{km}^{-1}$. Orange-pink colors indicate the presence of clouds; (b) color code for CI. Depending on the altitude, CI values below 2 to 5 (colors from grey to pink) indicate the presence of clouds. Median tropopause (TP $\left.{ }_{\text {med }}\right)$ and the percentile 95 of the tropopause $\left(\mathrm{TP}_{95}\right)$ are represented with orange and yellow circles, respectively. Cloud top height $(\mathrm{CTH})$ and cloud bottom height $(\mathrm{CBH})$ are represented with a black triangle and with a white circle, respectively. The altitude of the tangent points (TgPt) is the $\mathrm{y}$ axis. The white areas in both cross-sections correspond to a first filtering of optically thicker regions $(\mathrm{CI}<2)$. 

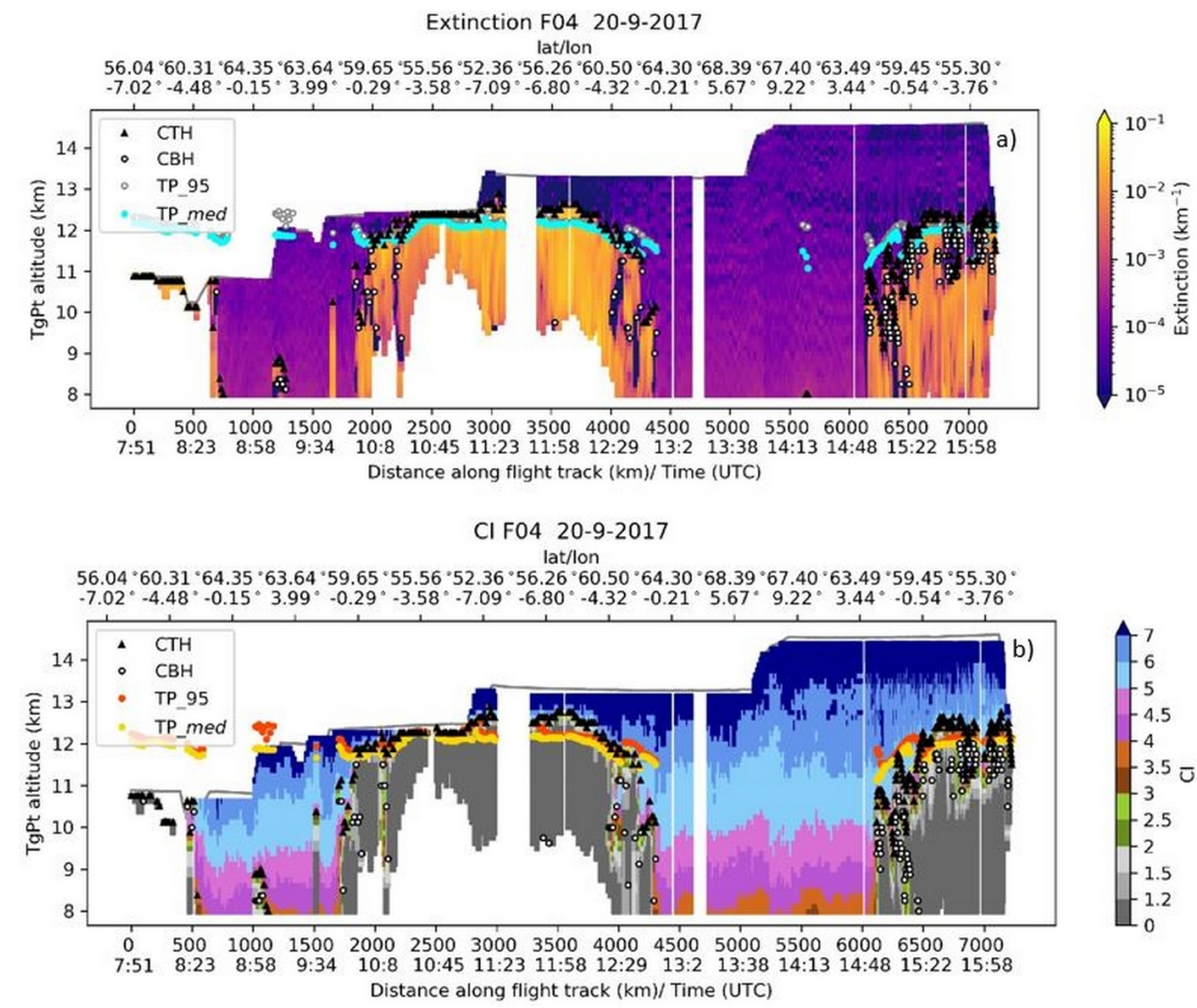

Figure S3. Cross-sections of extinction (a) and cloud index (b) for flight 4 of the WISE campaign. The results are restricted to levels below flight path. (a) Color code for extinction in $\mathrm{km}^{-1}$. Orange-pink colors indicate the presence of clouds; (b) color code for CI. Depending on the altitude, CI values below 2 to 5 (colors from grey to pink) indicate the presence of clouds. Median tropopause (TPmed) and the percentile 95 of the tropopause $\left(\mathrm{TP}_{95}\right)$ are represented with orange and yellow circles, respectively. Cloud top height $(\mathrm{CTH})$ and cloud bottom height $(\mathrm{CBH})$ are represented with a black triangle and with a white circle, respectively. The altitude of the tangent points $(\mathrm{TgPt})$ is the $\mathrm{y}$ axis. The white areas in both cross-sections correspond to a first filtering of optically thicker regions $(\mathrm{CI}<2)$. 


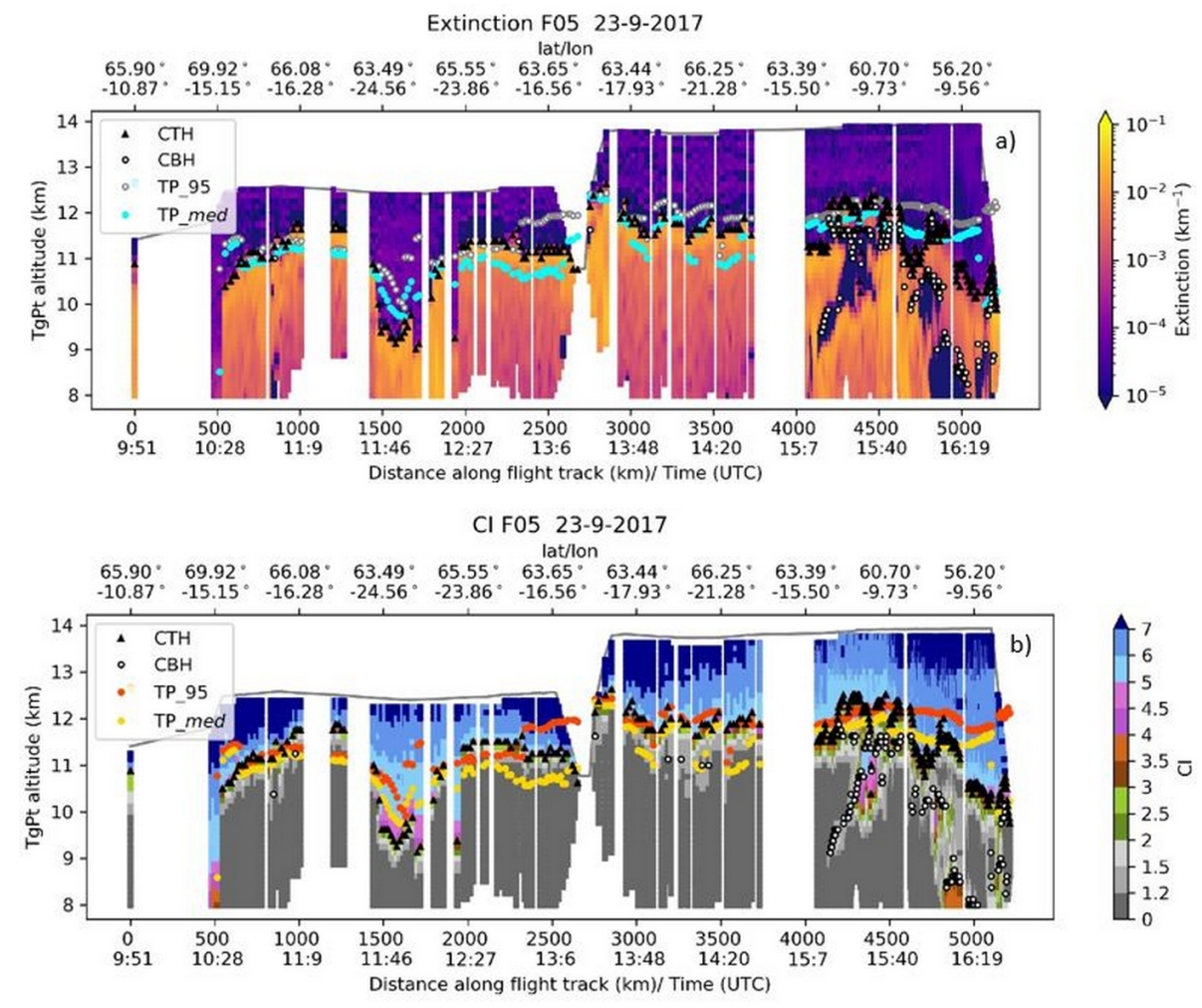

Figure S4. Cross-sections of extinction (a) and cloud index (b) for flight 5 of the WISE campaign. The results are restricted to levels below flight path. (a) Color code for extinction in $\mathrm{km}^{-1}$. Orange-pink colors indicate the presence of clouds; (b) color code for CI. Depending on the altitude, CI values below 2 to 5 (colors from grey to pink) indicate the presence of clouds. Median tropopause (TP $\mathrm{med})$ and the percentile 95 of the tropopause $\left(\mathrm{TP}_{95}\right)$ are represented with orange and yellow circles, respectively. Cloud top height $(\mathrm{CTH})$ and cloud bottom height $(\mathrm{CBH})$ are represented with a black triangle and with a white circle, respectively. The altitude of the tangent points (TgPt) is the $\mathrm{y}$ axis. The white areas in both cross-sections correspond to a first filtering of optically thicker regions $(\mathrm{CI}<2)$. 

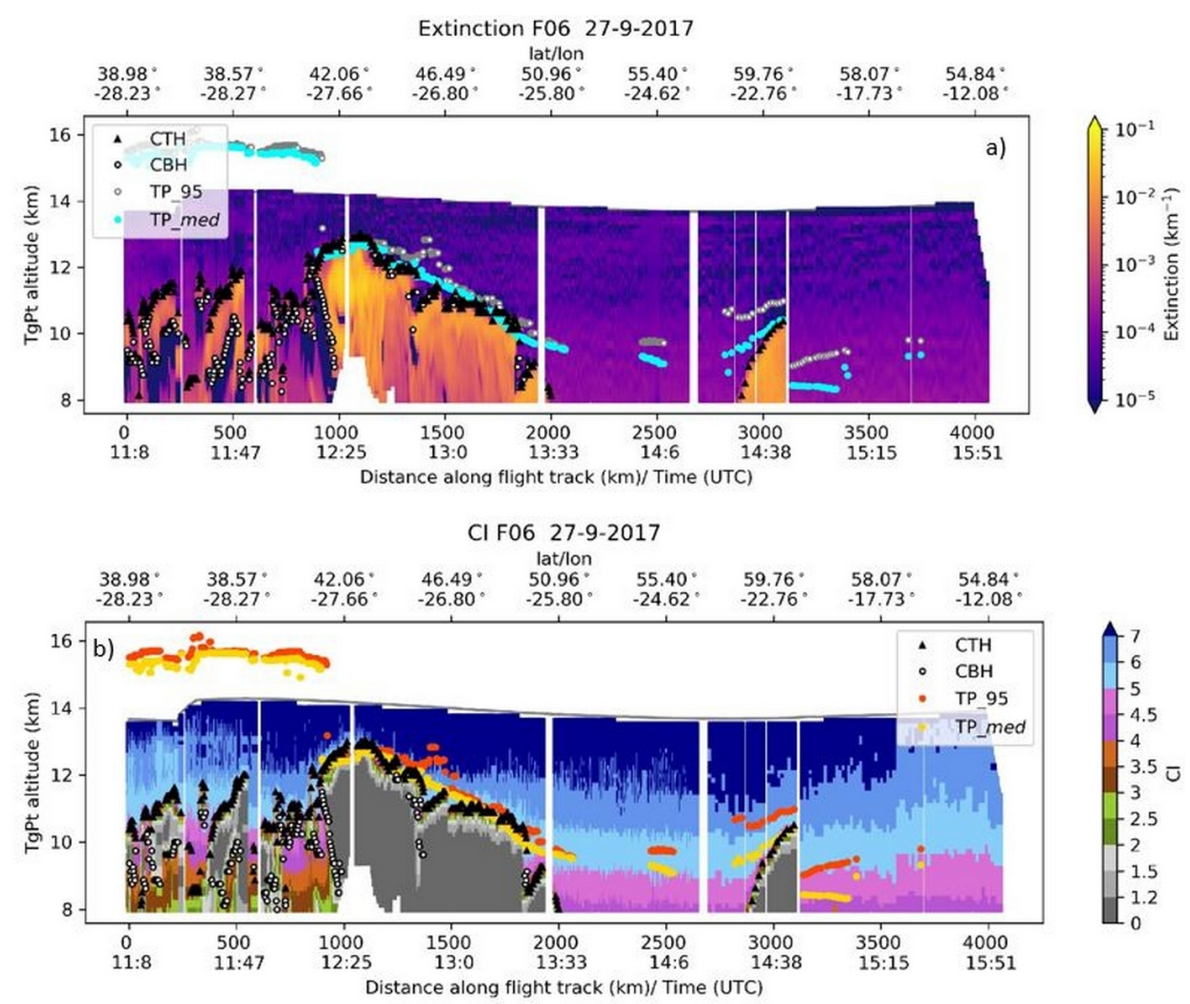

Figure S5. Cross-sections of extinction (a) and cloud index (b) for flight 6 of the WISE campaign. The results are restricted to levels below flight path. (a) Color code for extinction in $\mathrm{km}^{-1}$. Orange-pink colors indicate the presence of clouds; (b) color code for CI. Depending on the altitude, $\mathrm{CI}$ values below 2 to 5 (colors from grey to pink) indicate the presence of clouds. Median tropopause $\left(\mathrm{TP}_{\text {med }}\right)$ and the percentile 95 of the tropopause $\left(\mathrm{TP}_{95}\right)$ are represented with orange and yellow circles, respectively. Cloud top height $(\mathrm{CTH})$ and cloud bottom height $(\mathrm{CBH})$ are represented with a black triangle and with a white circle, respectively. The altitude of the tangent points (TgPt) is the y axis. The white areas in both cross-sections correspond to a first filtering of optically thicker regions $(\mathrm{CI}<2)$. 


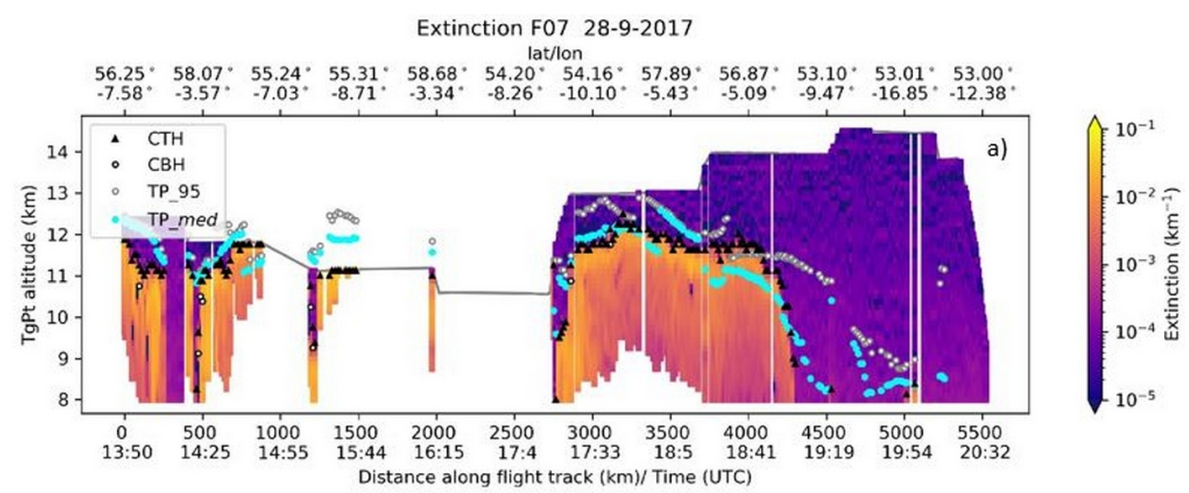

CI F07 28-9-2017

lat/lon

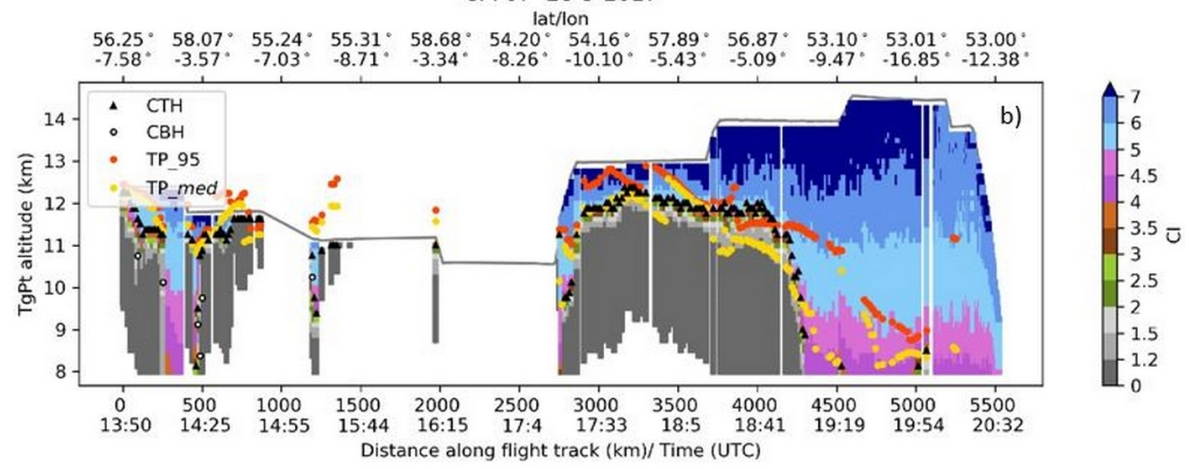

Figure S6. Cross-sections of extinction (a) and cloud index (b) for flight 7 of the WISE campaign. The results are restricted to levels below flight path. (a) Color code for extinction in $\mathrm{km}^{-1}$. Orange-pink colors indicate the presence of clouds; (b) color code for CI. Depending on the altitude, $\mathrm{CI}$ values below 2 to 5 (colors from grey to pink) indicate the presence of clouds. Median tropopause ( $\left.\mathrm{TP}_{\mathrm{med}}\right)$ and the percentile 95 of the tropopause $\left(\mathrm{TP}_{95}\right)$ are represented with orange and yellow circles, respectively. Cloud top height $(\mathrm{CTH})$ and cloud bottom height $(\mathrm{CBH})$ are represented with a black triangle and with a white circle, respectively. The altitude of the tangent points (TgPt) is the y axis. The white areas in both cross-sections correspond to a first filtering of optically thicker regions $(\mathrm{CI}<2)$. 


\section{Extinction F08 1-10-2017}

lat/lon

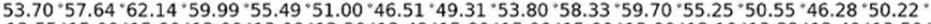

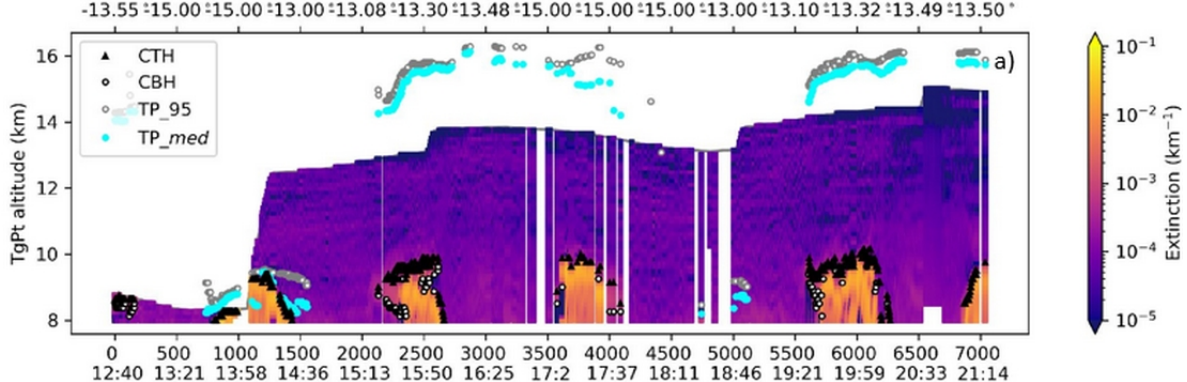

Distance along flight track ( $\mathrm{km}) /$ Time (UTC)

Cl F08 1-10-2017

lat/lon

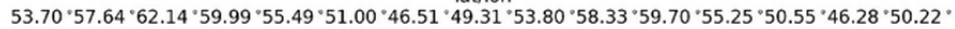

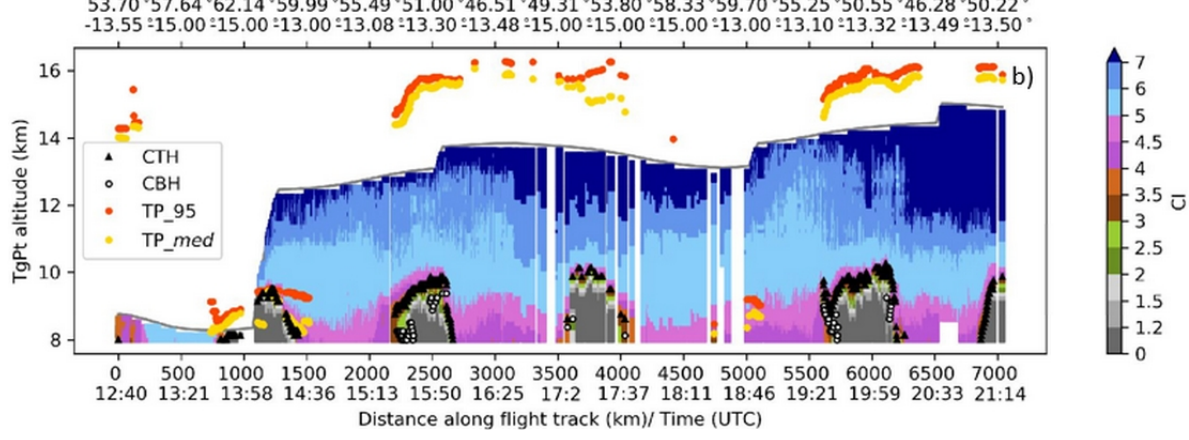

Figure S7. Cross-sections of extinction (a) and cloud index (b) for flight 8 of the WISE campaign. The results are restricted to levels below flight path. (a) Color code for extinction in $\mathrm{km}^{-1}$. Orange-pink colors indicate the presence of clouds; (b) color code for CI. Depending on the altitude, CI values below 2 to 5 (colors from grey to pink) indicate the presence of clouds. Median tropopause $\left(\mathrm{TP}_{\text {med }}\right)$ and the percentile 95 of the tropopause $\left(\mathrm{TP}_{95}\right)$ are represented with orange and yellow circles, respectively. Cloud top height (CTH) and cloud bottom height $(\mathrm{CBH})$ are represented with a black triangle and with a white circle, respectively. The altitude of the tangent points (TgPt) is the y axis. The white areas in both cross-sections correspond to a first filtering of optically thicker regions $(\mathrm{CI}<2)$. 
Extinction F09 4-10-2017

lat/lon

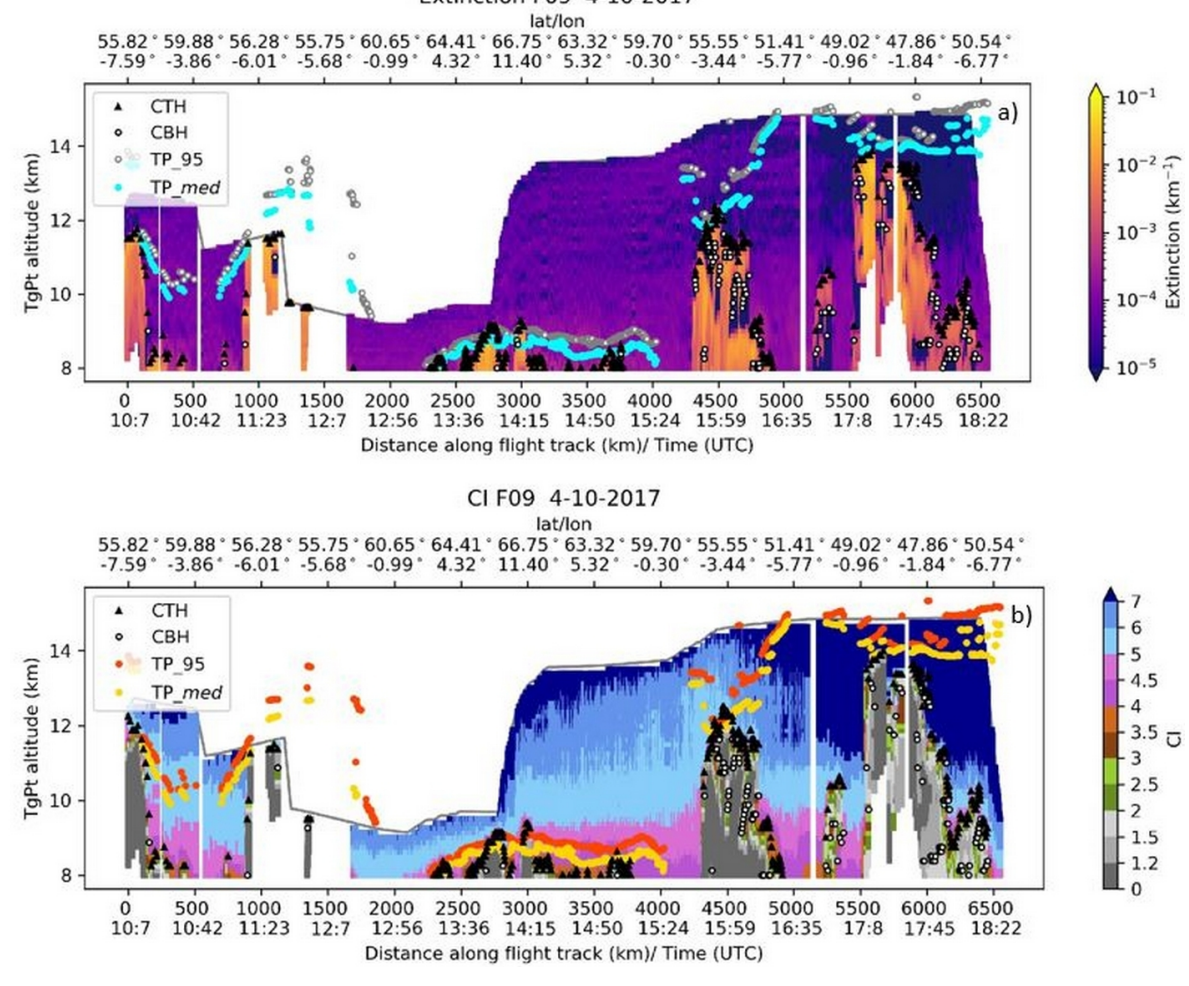

Figure S8. Cross-sections of extinction (a) and cloud index (b) for flight 9 of the WISE campaign. The results are restricted to levels below flight path. (a) Color code for extinction in $\mathrm{km}^{-1}$. Orange-pink colors indicate the presence of clouds; (b) color code for CI. Depending on the altitude, CI values below 2 to 5 (colors from grey to pink) indicate the presence of clouds. Median tropopause $\left(\mathrm{TP}_{\text {med }}\right)$ and the percentile 95 of the tropopause $\left(\mathrm{TP}_{95}\right)$ are represented with orange and yellow circles, respectively. Cloud top height (CTH) and cloud bottom height $(\mathrm{CBH})$ are represented with a black triangle and with a white circle, respectively. The altitude of the tangent points (TgPt) is the y axis. The white areas in both cross-sections correspond to a first filtering of optically thicker regions $(\mathrm{CI}<2)$. 
Extinction F10 7-10-2017

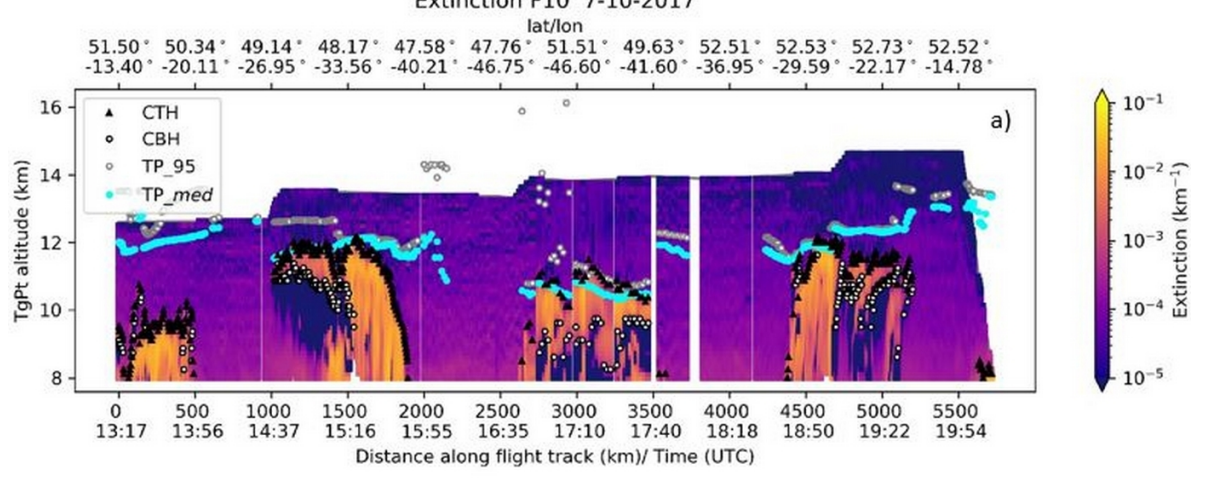

CI F10 7-10-2017 lat/lon

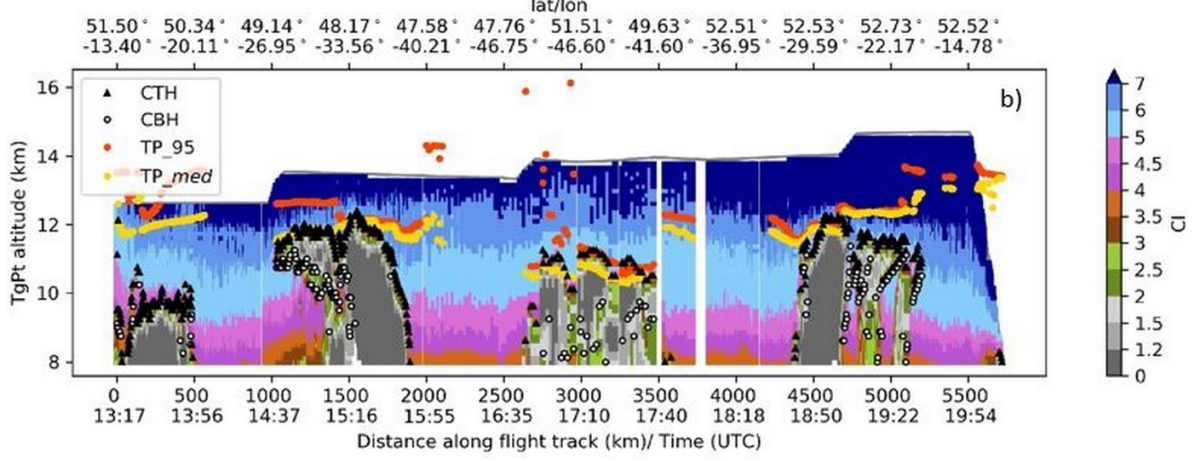

Figure S9. Cross-sections of extinction (a) and cloud index (b) for flight 10 of the WISE campaign. The results are restricted to levels below flight path. (a) Color code for extinction in $\mathrm{km}^{-1}$. Orange-pink colors indicate the presence of clouds; (b) color code for CI. Depending on the altitude, CI values below 2 to 5 (colors from grey to pink) indicate the presence of clouds. Median tropopause $\left(\mathrm{TP}_{\text {med }}\right)$ and the percentile 95 of the tropopause $\left(\mathrm{TP}_{95}\right)$ are represented with orange and yellow circles, respectively. Cloud top height (CTH) and cloud bottom height $(\mathrm{CBH})$ are represented with a black triangle and with a white circle, respectively. The altitude of the tangent points (TgPt) is the y axis. The white areas in both cross-sections correspond to a first filtering of optically thicker regions $(\mathrm{CI}<2)$. 
Extinction F11 9-10-2017

lat/lon

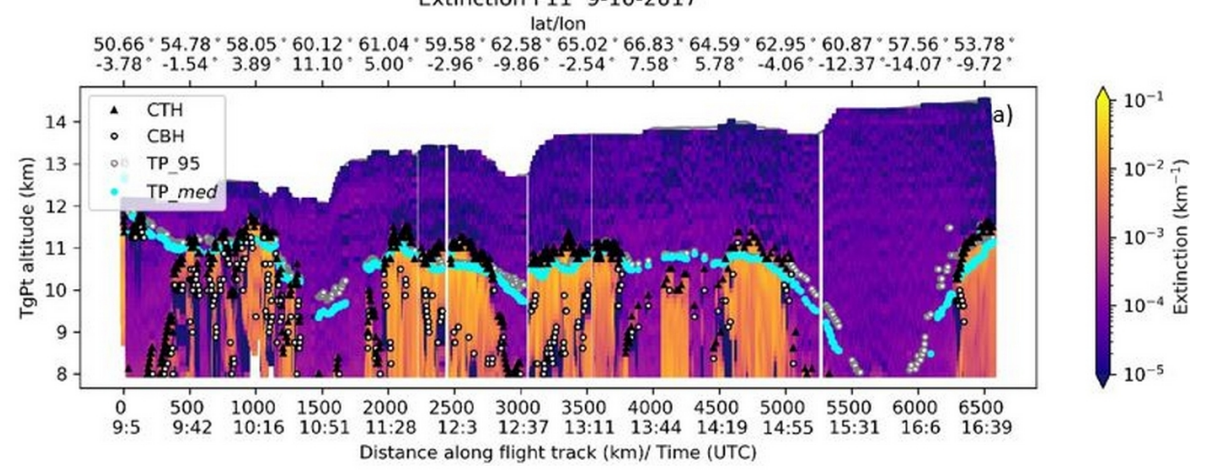

Cl F11 9-10-2017

lat/lon

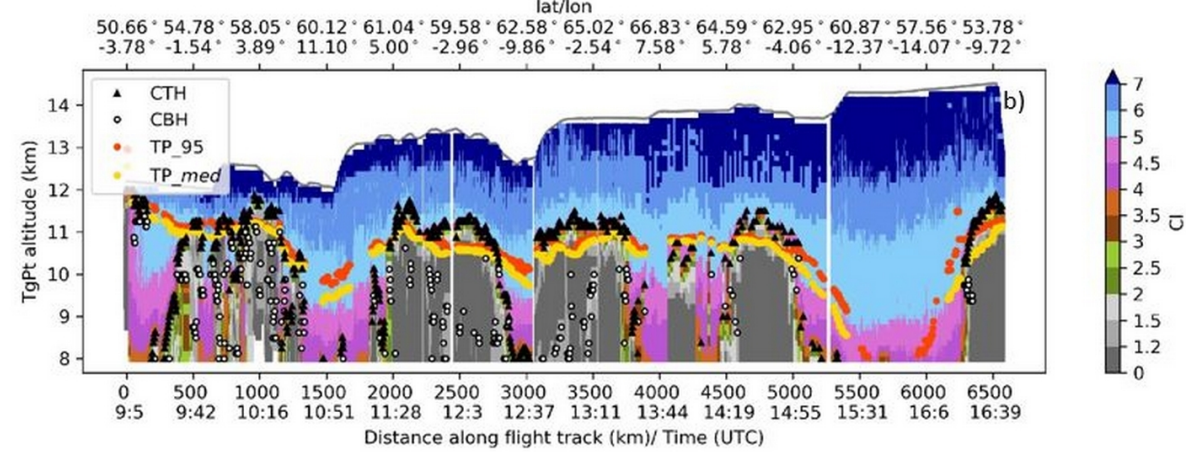

Figure S10. Cross-sections of extinction (a) and cloud index (b) for flight 11 of the WISE campaign. The results are restricted to levels below flight path. (a) Color code for extinction in $\mathrm{km}^{-1}$. Orange-pink colors indicate the presence of clouds; (b) color code for CI. Depending on the altitude, CI values below 2 to 5 (colors from grey to pink) indicate the presence of clouds. Median tropopause (TP $\mathrm{med}_{\text {) }}$ and the percentile 95 of the tropopause $\left(\mathrm{TP}_{95}\right)$ are represented with orange and yellow circles, respectively. Cloud top height (CTH) and cloud bottom height $(\mathrm{CBH})$ are represented with a black triangle and with a white circle, respectively. The altitude of the tangent points (TgPt) is the $\mathrm{y}$ axis. The white areas in both cross-sections correspond to a first filtering of optically thicker regions $(\mathrm{CI}<2)$. 


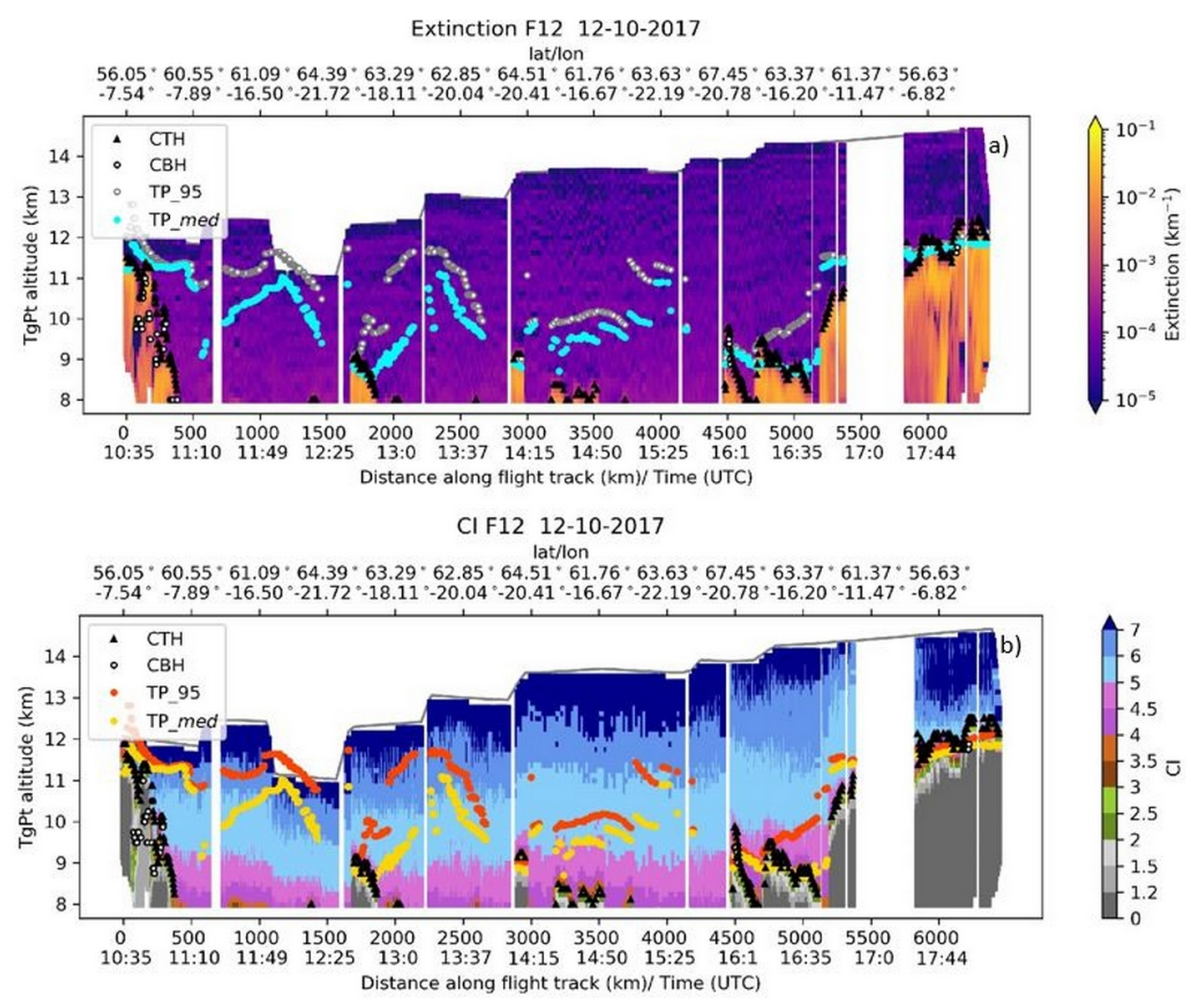

Figure S11. Cross-sections of extinction (a) and cloud index (b) for flight 12 of the WISE campaign. The results are restricted to levels below flight path. (a) Color code for extinction in $\mathrm{km}^{-1}$. Orange-pink colors indicate the presence of clouds; (b) color code for CI. Depending on the altitude, CI values below 2 to 5 (colors from grey to pink) indicate the presence of clouds. Median tropopause $\left(\mathrm{TP}_{\mathrm{med}}\right)$ and the percentile 95 of the tropopause $\left(\mathrm{TP}_{95}\right)$ are represented with orange and yellow circles, respectively. Cloud top height (CTH) and cloud bottom height $(\mathrm{CBH})$ are represented with a black triangle and with a white circle, respectively. The altitude of the tangent points $(\mathrm{TgPt})$ is the $\mathrm{y}$ axis. The white areas in both cross-sections correspond to a first filtering of optically thicker regions $(\mathrm{CI}<2)$. 
Extinction F13 14-10-2017

lat/lon

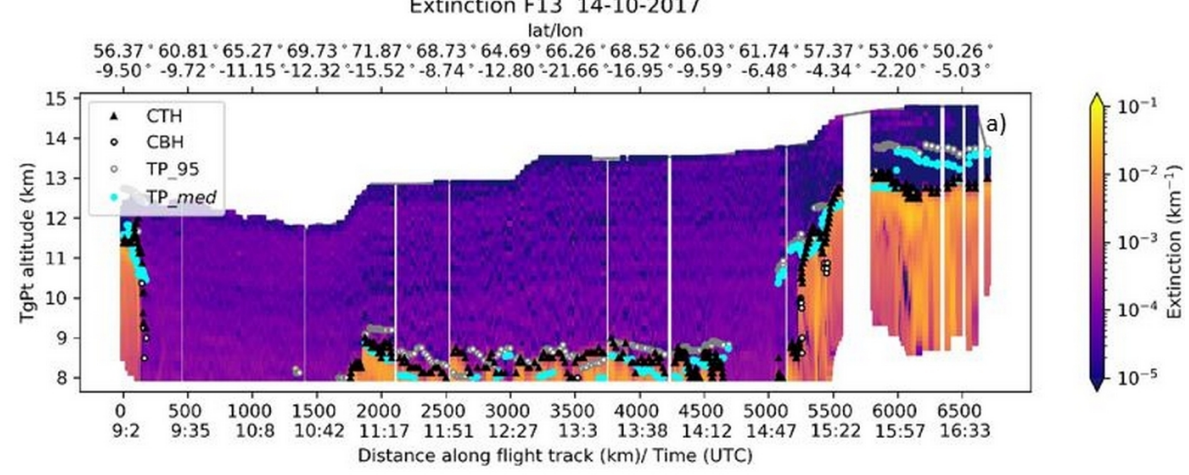

Cl F13 14-10-2017

lat/lon

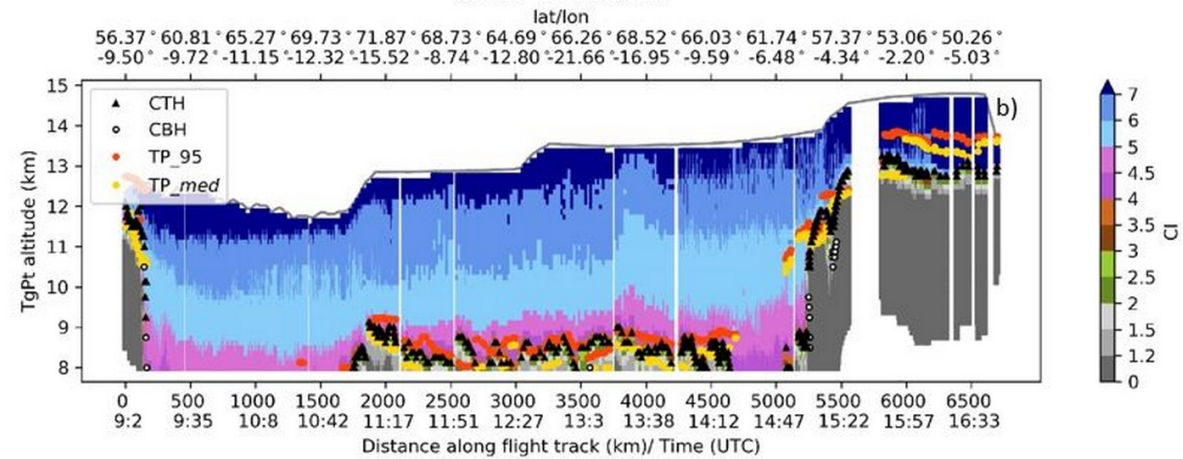

Figure S12. Cross-sections of extinction (a) and cloud index (b) for flight 13 of the WISE campaign. The results are restricted to levels below flight path. (a) Color code for extinction in $\mathrm{km}^{-1}$. Orange-pink colors indicate the presence of clouds; (b) color code for CI. Depending on the altitude, $\mathrm{CI}$ values below 2 to 5 (colors from grey to pink) indicate the presence of clouds. Median tropopause $\left(\mathrm{TP}_{\mathrm{med}}\right)$ and the percentile 95 of the tropopause $\left(\mathrm{TP}_{95}\right)$ are represented with orange and yellow circles, respectively. Cloud top height $(\mathrm{CTH})$ and cloud bottom height $(\mathrm{CBH})$ are represented with a black triangle and with a white circle, respectively. The altitude of the tangent points (TgPt) is the y axis. The white areas in both cross-sections correspond to a first filtering of optically thicker regions $(\mathrm{CI}<2)$. 

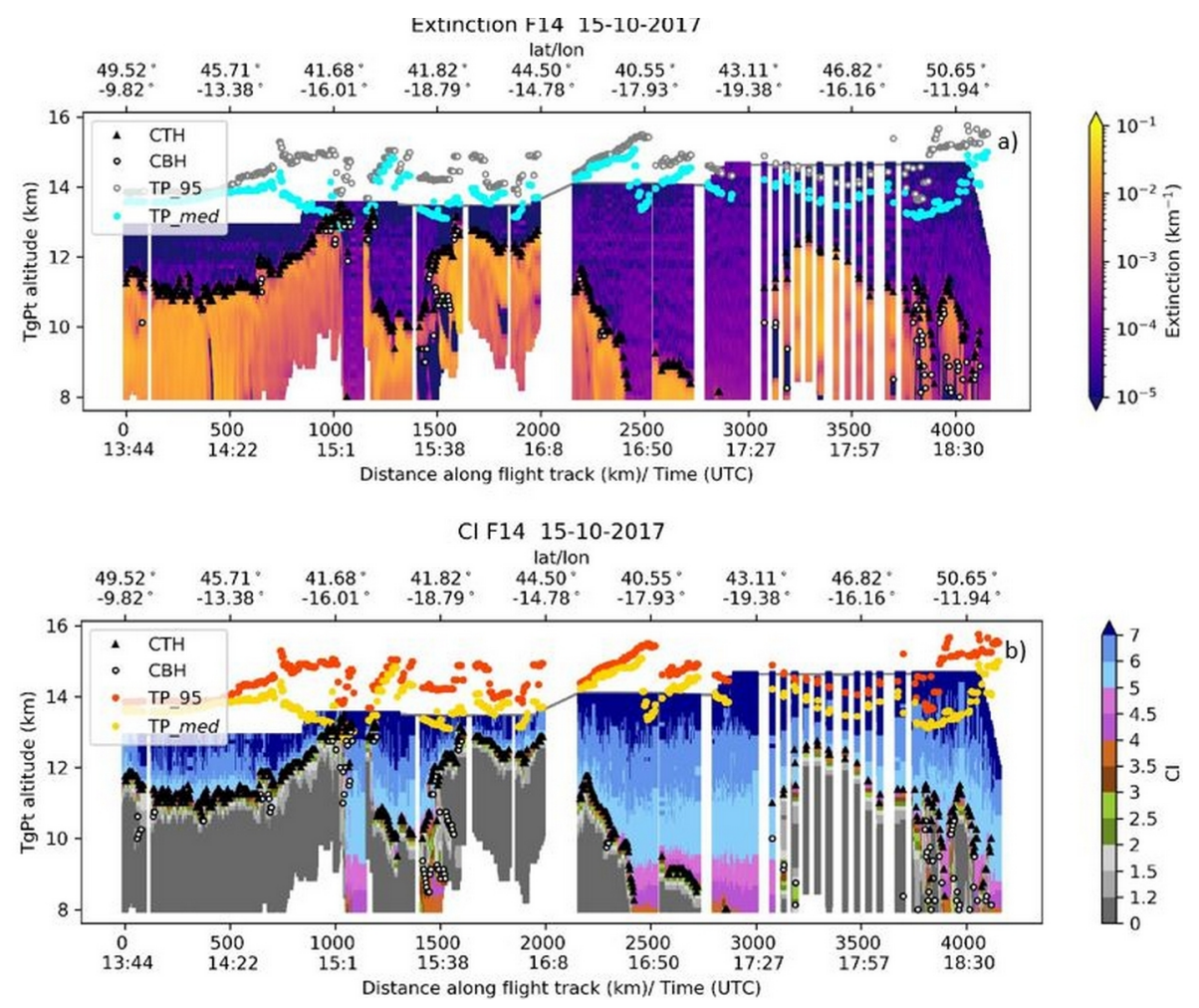

Figure S13. Cross-sections of extinction (a) and cloud index (b) for flight 14 of the WISE campaign. The results are restricted to levels below flight path. (a) Color code for extinction in $\mathrm{km}^{-1}$. Orange-pink colors indicate the presence of clouds; (b) color code for CI. Depending on the altitude, CI values below 2 to 5 (colors from grey to pink) indicate the presence of clouds. Median tropopause (TP $\left.{ }_{\text {med }}\right)$ and the percentile 95 of the tropopause $\left(\mathrm{TP}_{95}\right)$ are represented with orange and yellow circles, respectively. Cloud top height $(\mathrm{CTH})$ and cloud bottom height $(\mathrm{CBH})$ are represented with a black triangle and with a white circle, respectively. The altitude of the tangent points (TgPt) is the $\mathrm{y}$ axis. The white areas in both cross-sections correspond to a first filtering of optically thicker regions $(\mathrm{CI}<2)$. 
lat/lon

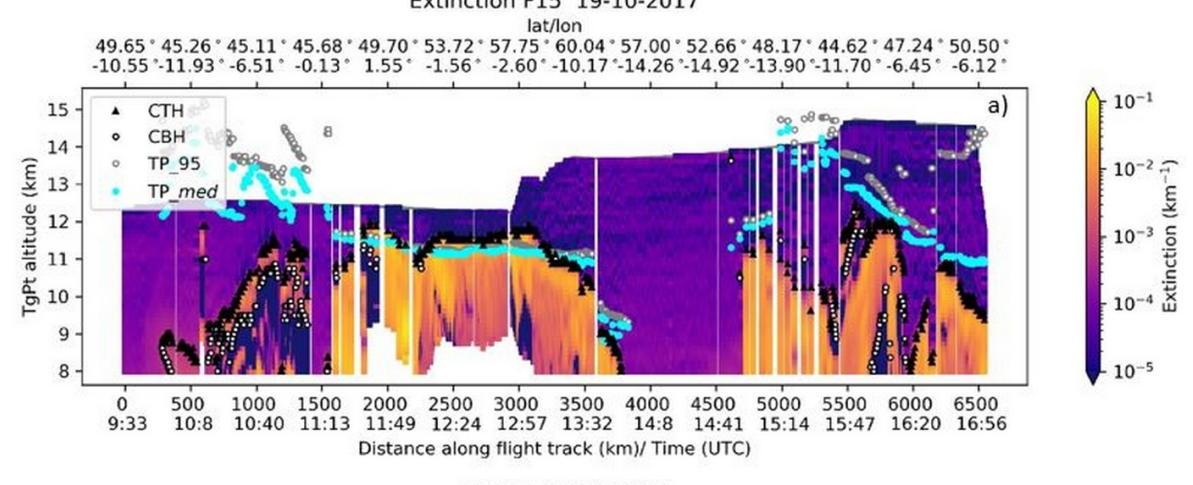

Cl F15 19-10-2017 lat/lon

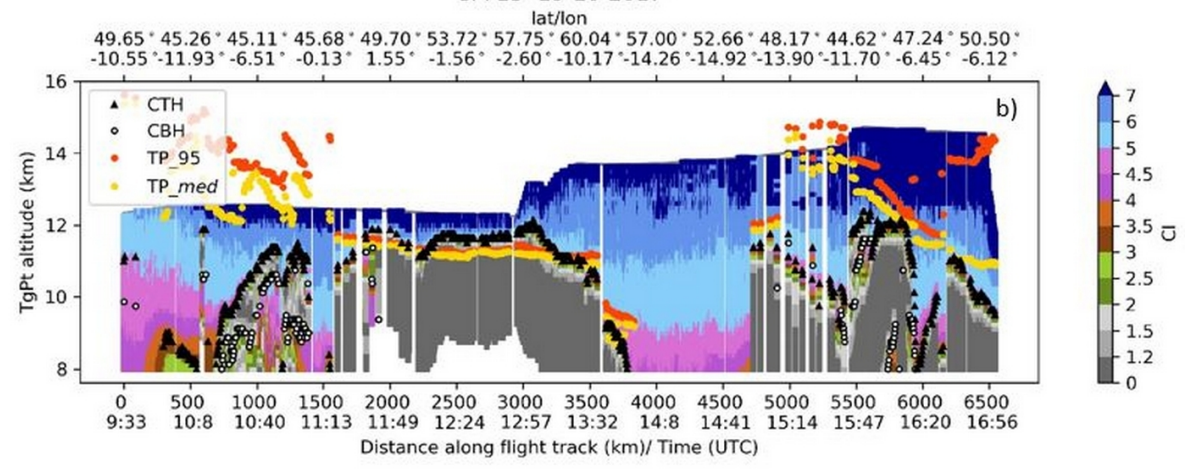

Figure S14. Cross-sections of extinction (a) and cloud index (b) for flight 15 of the WISE campaign. The results are restricted to levels below flight path. (a) Color code for extinction in $\mathrm{km}^{-1}$. Orange-pink colors indicate the presence of clouds; (b) color code for CI. Depending on the altitude, $\mathrm{CI}$ values below 2 to 5 (colors from grey to pink) indicate the presence of clouds. Median tropopause ( $\left.\mathrm{TP}_{\mathrm{med}}\right)$ and the percentile 95 of the tropopause $\left(\mathrm{TP}_{95}\right)$ are represented with orange and yellow circles, respectively. Cloud top height (CTH) and cloud bottom height $(\mathrm{CBH})$ are represented with a black triangle and with a white circle, respectively. The altitude of the tangent points ( $\mathrm{TgPt})$ is the y axis. The white areas in both cross-sections correspond to a first filtering of optically thicker regions $(\mathrm{CI}<2)$. 

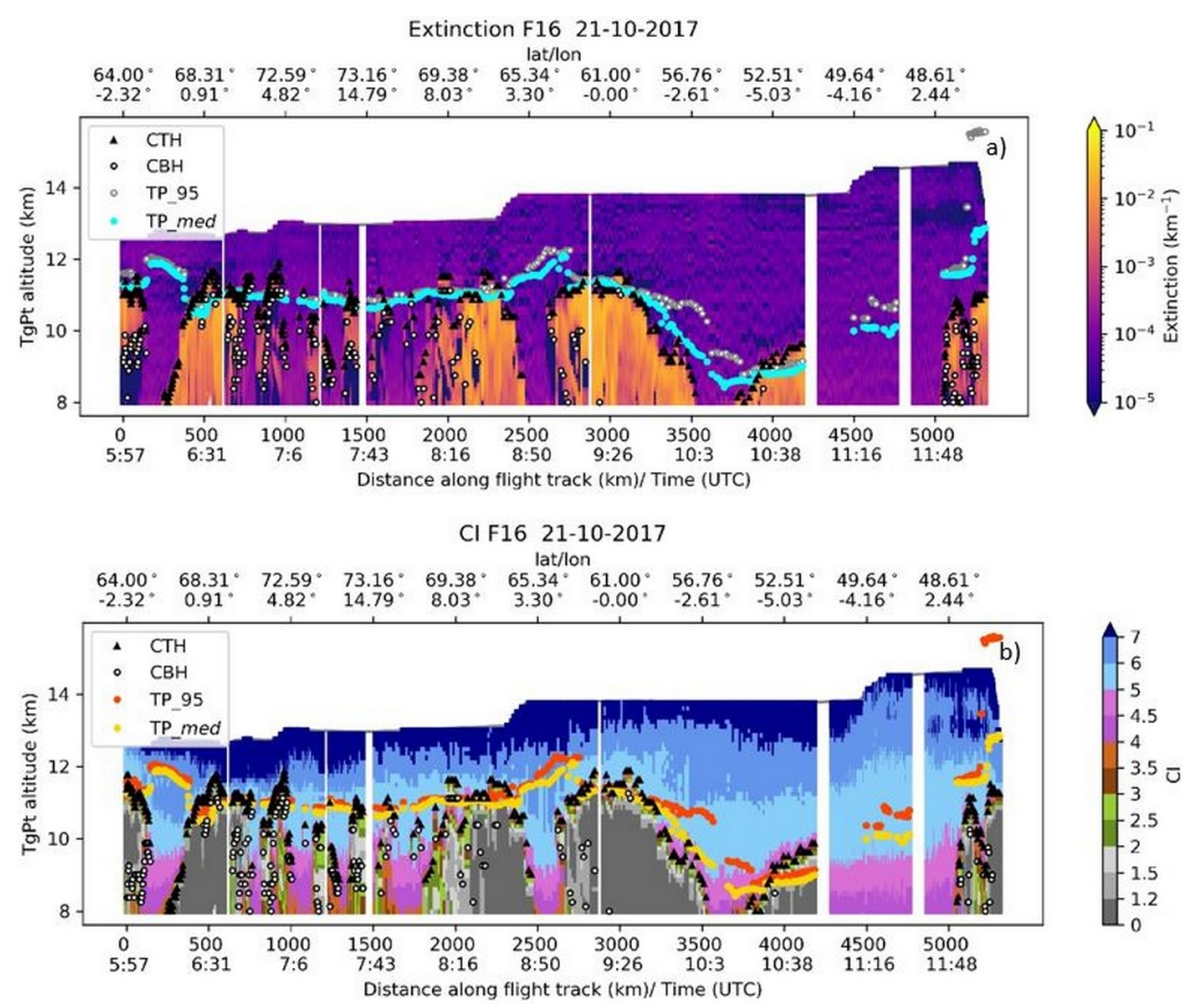

Figure S15. Cross-sections of extinction (a) and cloud index (b) for flight 16 of the WISE campaign. The results are restricted to levels below flight path. (a) Color code for extinction in $\mathrm{km}^{-1}$. Orange-pink colors indicate the presence of clouds; (b) color code for CI. Depending on the altitude, CI values below 2 to 5 (colors from grey to pink) indicate the presence of clouds. Median tropopause $\left(\mathrm{TP}_{\text {med }}\right)$ and the percentile 95 of the tropopause $\left(\mathrm{TP}_{95}\right)$ are represented with orange and yellow circles, respectively. Cloud top height $(\mathrm{CTH})$ and cloud bottom height $(\mathrm{CBH})$ are represented with a black triangle and with a white circle, respectively. The altitude of the tangent points (TgPt) is the $\mathrm{y}$ axis. The white areas in both cross-sections correspond to a first filtering of optically thicker regions $(\mathrm{CI}<2)$. 